\title{
Multifractal analysis of the branch structure of diffusion-limited aggregates
}

\author{
W. G. Hanan and D. M. Heffernan \\ Department of Mathematical Physics, National University of Ireland Maynooth, County Kildare, Ireland
}

(Received 3 November 2011; published 27 February 2012)

\begin{abstract}
We examine the branch structure of radial diffusion-limited aggregation (DLA) clusters for evidence of multifractality. The lacunarity of DLA clusters is measured and the generalized dimensions $D(q)$ of their mass distribution is estimated using the sandbox method. We find that the global $n$-fold symmetry of the aggregates can induce anomalous scaling behavior into these measurements. However, negating the effects of this symmetry, standard scaling is recovered.
\end{abstract}

DOI: 10.1103/PhysRevE.85.021407

PACS number(s): 61.43.Hv, 05.45.Df

\section{INTRODUCTION}

Diffusion-limited aggregation (DLA) is a stochastic growth model that produces fractal clusters via the release of randomly walking particles that adhere irreversibly to the growing aggregate on first contact [1]. The resultant clusters possess a characteristic dendritic structure, the growth of which is determined by a Laplacian field [2]. Similar Laplacian growth processes are ubiquitous in nature so that DLA-like patterns are observed in a diverse range of seemingly unrelated phenomena. Such patterns can be seen, for example, in fluid-fluid displacement experiments in a Hele-Shaw cell, in the discharge pattern created inside an insulator exposed to a large electric field, and in the growth of bacterial colonies grown at low nutrient concentrations [3].

The generality and simplicity of the DLA model has thus seen it play a paradigmatic role in the field of kinetic growth phenomena. Soon after the introduction of the model in 1981, it was discovered that the growth probability along the cluster boundary was multifractal [4-7]. The growth probability distribution thus required an infinite hierarchy of dimensions $D(q)$ for its scaling properties to be fully characterized $[8,9]$. This in turn led to speculation that the dendritic branch structure of the clusters themselves might also be multifractal $[10,11]$.

Initial numerical evaluations of the $D(q)$ spectrum of the mass distribution seemed to suggest that such speculation was misguided and that DLA was a simple monofractal with constant $D(q)$ [12-14]. However, there quickly followed contrary work that supported the multifractal scenario $[11,15,16]$. To our knowledge, there has been little if any direct research on this subject performed since, and the possible multifractality of DLA branch structure thus still remains unresolved [17].

In this paper, we revisit this issue by examining some of the numerical evidence originally put forward in support of multifractality. Replicating some of these earlier investigations, we measure the lacunarity and $D(q)$ spectrum of radial DLA clusters and find that the case for multifractality is not as strong as previously thought. We show that the anomalous scaling originally attributed as evidence of multifractality is in fact a numerical artifact induced by the global $n$-fold symmetry of the clusters. In Fig. 1, this symmetry is easily identified by the approximately seven principal cluster arms, which emanate radially outward from the central cluster seed point [18].

We demonstrate, however, that if one can negate the effects of this $n$-fold symmetry on the numerical results, one can ultimately uncover the scaling properties of the fractal branch structure, which lies within these principal arms.

\section{NUMERICAL RESULTS}

Some fractal objects require only a single exponent, the fractal dimension, to fully characterize the self-similar properties of their mass distribution (or Lebesque measure) $[9,14,19]$. Such objects are thus often referred to as simple fractals or monofractals, the middle third Cantor set being one commonly known example. However, more complicated objects require an infinite hierarchy of exponents to be described. In such cases, the object is said to be multifractal and is usually characterized by either providing the generalized spectrum of dimensions $D(q)$ or, equivalently, the $f(\alpha)$ spectrum [8,9]. While, for multifractals, both $D(q)$ and $f(\alpha)$ are by definition nontrivial in form, for monofractals, $D(q)$ is constant for all $q$ with the associated $f(\alpha)$ function being reduced to a single point [9].

Later in this section, we shall therefore be performing a direct estimation of $D(q)$ on the mass distribution of radial DLA clusters in an attempt to address the question of their possible multifractality. Before doing so, however, we discuss the lacunarity of such clusters.

\section{A. Lacunarity}

In addition to the fractal dimension, the lacunarity of fractal objects is often calculated as a means of further characterizing them [20]. In essence, lacunarity is simply a measure of the degree of homogeneity in the mass distribution of an object. Let us take as our object, for example, a radial DLA cluster with its seed point located at the origin. Choosing a random cluster point located at some position $\mathbf{b}$, henceforth referred to as a reference point, we can calculate the Lebesque measure $\mu_{\mathbf{b}}(r)$ contained within a circle of radius $r$ centered on this point. Note that here we are defining the Lebesque measure $\mu_{\mathbf{b}}(r)$ to be simply the fraction of cluster points contained within the circle.

For fixed $r$, the quantity $\mu_{\mathbf{b}}(r)$ will thus necessarily vary with the location $\mathbf{b}$ of the reference point. Calculating its mean $\left\langle\mu_{\mathbf{b}}(r)\right\rangle$ and second moment $\left\langle\mu_{\mathbf{b}}^{2}(r)\right\rangle$, we may define the lacunarity of the cluster to be [20]

$$
\Phi(r)=\frac{\left\langle\mu_{\mathbf{b}}^{2}(r)\right\rangle}{\left\langle\mu_{\mathbf{b}}(r)\right\rangle^{2}}=1+\frac{\sigma^{2}(r)}{\left\langle\mu_{\mathbf{b}}(r)\right\rangle^{2}},
$$




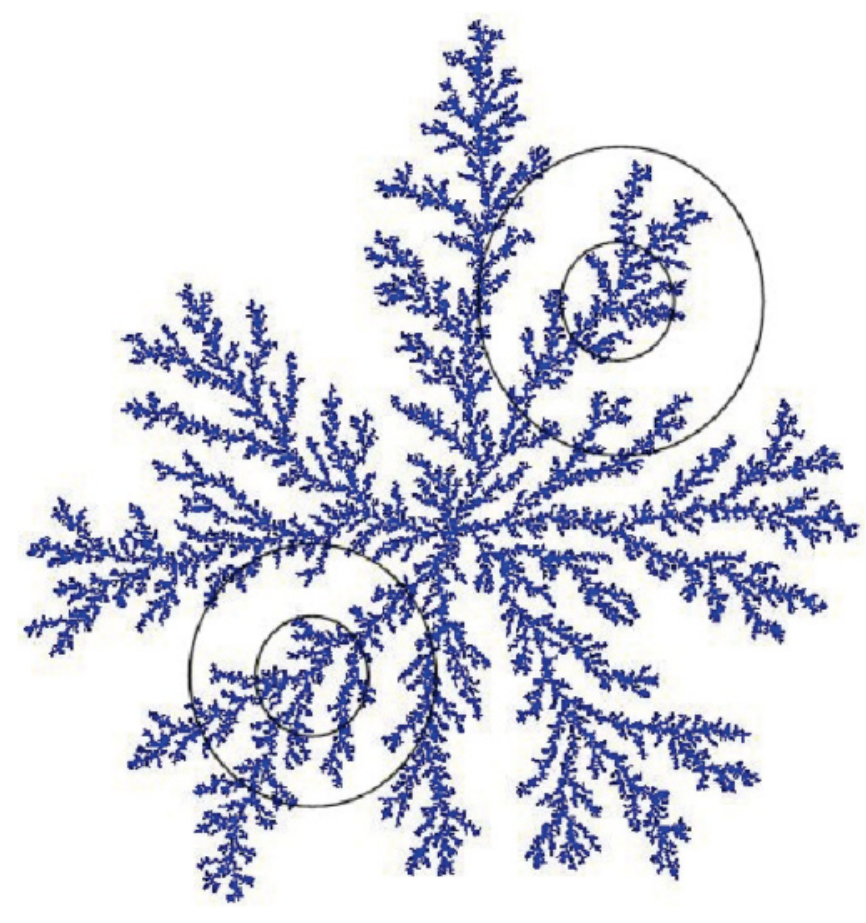

FIG. 1. (Color online) Radial DLA cluster of 100000 particles. Two reference points are chosen from the cluster. The inner circles show when boundary effects are expected to set in as empty space begins to be sampled. As the circles grow larger, at some point (shown by the outer circles), they will crash sideways into the neighboring cluster arms.

where $\sigma^{2}(r)$ is the variance in $\mu_{\mathbf{b}}(r)$. As $\sigma^{2}(r) \geqslant 0$, we immediately see that we must have $\Phi \geqslant 1$. Furthermore, if the structure is highly irregular in form, the variance $\sigma^{2}(r)$ will necessarily have a large value and the lacunarity $\Phi$ will thus also be correspondingly large. Alternatively, a more homogeneous and regular structure will yield a value of $\Phi$ closer to 1 (see Fig. 11).

For a monofractal object, we expect $\Phi$ to be constant over length scales where the object is self-similar. This is simply because the structure appears unchanged in appearance over these scales and, as the mass is distributed in a homogeneous fashion, the relative spread $\sigma(r) /\left\langle\mu_{\mathbf{b}}(r)\right\rangle$ in $\mu_{\mathbf{b}}(r)$ is consequently invariant with $r$. Growing 100 off-lattice radial DLA clusters up to a mass of $10^{6}$ particles, we measured $\Phi$ as a function of $r$. We did this by initially evaluating the averages in Eq. (1) using only reference points lying within a distance $0.5 R_{g}$ of the cluster seed point, where $R_{g}$ is the radius of gyration of an individual cluster. This ensures that we only sample the frozen core and not the still incomplete structure within the "active zone" of the clusters [15,21].

The numerical results are displayed in Fig. 2(a), where we also show $\Phi(r)$ curves evaluated at earlier stages in the growth of the clusters. (Note that the lacunarity values in the graphs of Fig. 2 actually denote the ensemble average of $\Phi$ taken over our 100 clusters.) As observed in previous studies, we see that $\Phi$ decreases dramatically with $r$ [15,22]. This suggests that the interior of radial DLA clusters is not monofractal in form. In fact, the observed decreasing lacunarity has in the past been used to support the case that the frozen zone of DLA clusters possesses a "complex internal structure," possibly synonymous with multifractality $[15,23]$.

To see why, note that for self-similar structure, the moments of the random variable $\mu_{\mathbf{b}}$ are expected to scale as [11]

$$
\left\langle\mu_{\mathbf{b}}^{q-1}(\epsilon)\right\rangle \sim \epsilon^{(q-1) D(q)},
$$

where $\epsilon=r / R_{g}$ and $q$ is any real value. Letting $q=2$ and 3, we thus find from (1) that

$$
\Phi(\epsilon) \sim \epsilon^{2[D(3)-D(2)]} .
$$

For a monofractal, $D(2)=D(3)$ and Eq. (3) thus predicts that $\Phi$ is independent of $r$ as mentioned previously. Alternatively, for a multifractal, $D(q)$ is necessarily a monotonically decreasing function of $q$ [8]. Consequently, we expect that $D(2)>D(3)$ and $\Phi$ should thus decrease as a power law with $r$. Although in Fig. 2(a), $\Phi$ certainly decreases with $r$, one can not, however, make a strong case that it does so in a power law fashion. It would appear therefore that while the data, as presented, clearly do not support the monofractal picture of DLA, neither is it convincing evidence for the multifractal scenario. However, note that when attempting to make such interpretations of the data, we are making one crucial implicit assumption. We are assuming that the measured lacunarity of Fig. 2(a) is solely characterizing the fractal structure of the DLA clusters. However, this is not the case.

Critically, the fractal structure we wish to characterize is defined by the self-similar dendritic branching that is found within the principal arms of our clusters. At relatively small $r$, the measure $\mu_{\mathbf{b}}(r)$ resides on cluster points belonging to the same principal cluster arm, and we are thus correctly probing the internal fractal structure. However, at larger $r$, we begin to sample a different structure, namely, the nonfractal $n$-fold symmetry of the aggregates. Because of this symmetry, the cluster points contributing to $\mu_{\mathbf{b}}(r)$ now come from more than one cluster arm and the sample values of $\mu_{\mathbf{b}}(r)$ become anomalously large and vary relatively little with position $\mathbf{b}$. At large $r \approx R_{g}$, we thus find that $\sigma /\left\langle\mu_{\mathbf{b}}\right\rangle \approx 0$ and the cluster subsequently appears homogeneous with a lacunarity close to unity. The decrease of $\log \Phi$ to zero in Fig. 2(a) is therefore merely a reflection of the $n$-fold symmetrical nature of the clusters.

If our aim is to examine purely the lacunarity of the internal fractal structure, we must thus avoid sampling the $n$-fold symmetry. Ideally, therefore, we should take each cluster, pluck the individual principal cluster arms from it, and then apply our algorithm as before on each such arm in isolation. An alternative, if less satisfactory method, is to simply take the samples of $\mu_{\mathbf{b}}(r)$ about reference points much further out from the seed point. To see why this helps, note that the linear distance between neighboring principal cluster arms increases as we move away from the seed. For a more outlying reference point, we therefore begin to sample the mass from neighboring principal cluster arms at larger values of $r$ than otherwise found for a reference point nearer the cluster seed. This thus opens up a wider range of length scales, which are now effectively free of the effects of the $n$-fold symmetry.

We therefore implemented our algorithm as before on our 100 DLA clusters, but calculated the averages in Eq. (1) only 

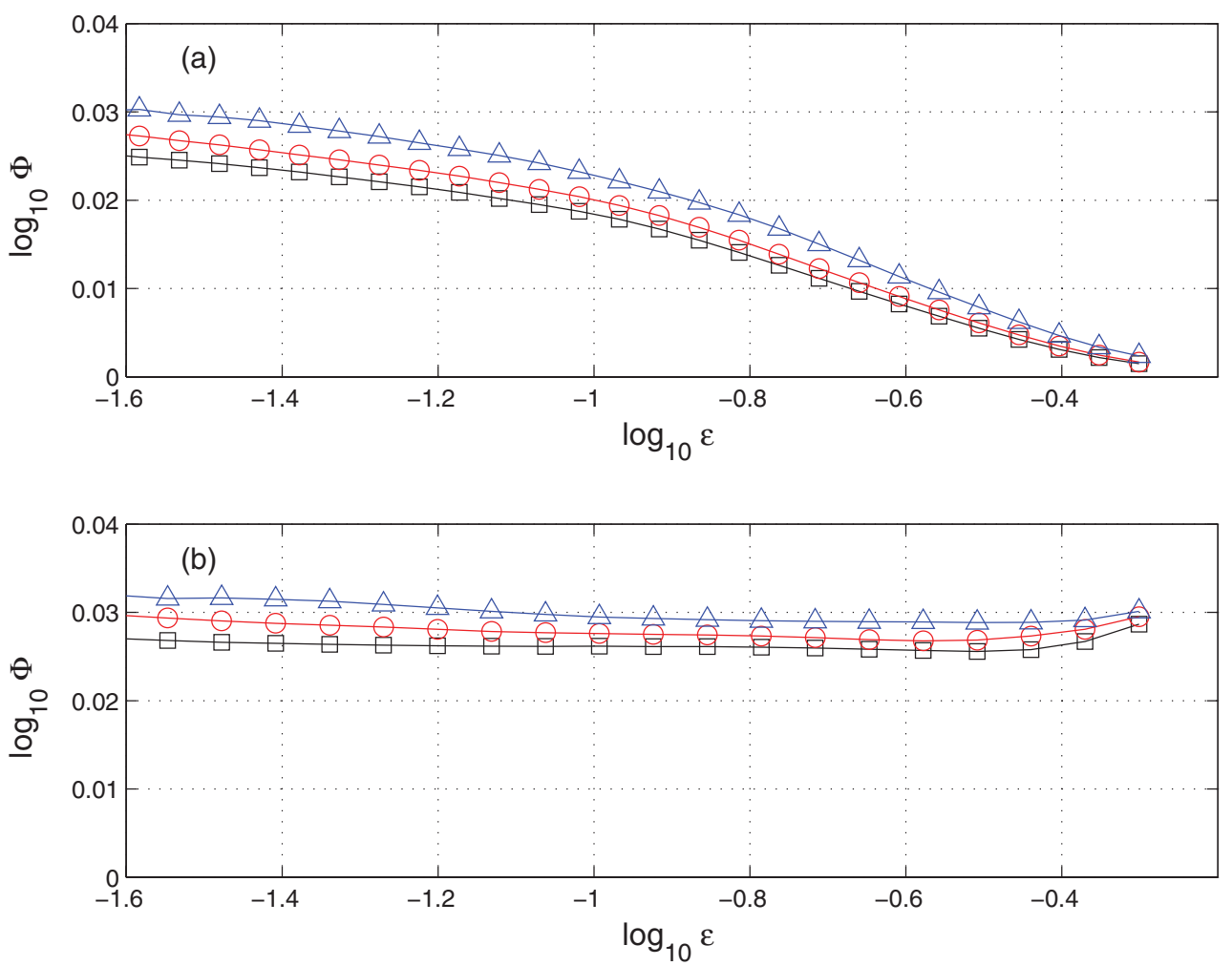

FIG. 2. (Color online) Lacunarity $\Phi$ of DLA as a function of $\epsilon=r / R_{g}$. Measured using 100 DLA clusters of mass $M=10^{6}$ ( $\square$ ), 125000 (०), and $15625(\triangle)$ particles and choosing only reference points that satisfy (a) $|\mathbf{b}|<0.5 R_{g}$ and (b) $0.5 R_{g}<|\mathbf{b}|<1.5 R_{g}$.

about reference points satisfying $0.5 R_{g}<|\mathrm{b}|<1.5 R_{g}$. The results from this procedure are shown in Fig. 2(b). We find that the dramatic decrease of $\Phi$ with $r$ no longer exists, thus demonstrating that, as suspected, this was merely a numerical artifact induced by the sampling in the frozen zone of the $n$-fold symmetry of the clusters. Instead, we now find that for a fixed cluster size, the lacunarity is essentially constant with $r$, a scenario consistent with the monofractal picture of DLA.

However, one obvious anomalous feature still remains. In both the data sets of Fig. 2, we observe that, for fixed $\epsilon$, the lacunarity $\Phi$ decreases with growth, behavior which is contrary to that expected of a self-similar object. We shall postpone discussion of this phenomenon until later in the paper. For now, we continue our investigation into the possible multifractality of DLA with a direct evaluation of the generalized spectrum of dimensions $D(q)$.

\section{B. Evaluation of $D(q)$}

In contrast to the numerous studies examining the growth probability measure of DLA [4-7,24-30], there have, to our knowledge, been very few direct estimates of the multifractal spectra characterizing the scaling properties of its mass distribution. And, those estimates that are available in the literature unfortunately give contradictory results. Some are consistent with a trivial $q$-independent monofractal form for $D(q)[13,14]$, while others support the multifractal scenario for DLA $[11,16]$.

In one of these latter studies, the frozen zone of off-lattice radial DLA clusters was analyzed and a multifractal $D(q)$ spectrum was obtained with $\Delta D=D(-\infty)-D(\infty) \approx 0.13$ [11]. This was done using the sandbox method [19], which involves the evaluation of $\left\langle\mu_{\mathbf{b}}^{q-1}(\epsilon)\right\rangle$ in Eq. (2). In this work, we implement the same procedure. By using the same ensemble of 100 DLA clusters of mass $M=10^{6}$ particles examined previously, we began by taking each cluster individually and chose 2000 random reference points from within $0.5 R_{g}$ of the seed point. For a fixed $\epsilon$, the sample average $\left\langle\mu_{\mathbf{b}}^{q-1}\right\rangle$ over these points was subsequently calculated for each cluster, before finally evaluating the ensemble average $\left\langle\log \left\langle\mu_{\mathbf{b}}^{q-1}\right\rangle\right\rangle$. This was done for various values of $q$ ranging from $q=-9 \rightarrow 9$ in steps of 0.06 .

From Eq. (2), plotting $\zeta_{q}=\frac{1}{(q-1)}\left\langle\log \left\langle\mu_{\mathbf{b}}^{q-1}\right\rangle\right\rangle$ against $\log \epsilon$ for some fixed $q$ should thus yield a straight line with slope $D(q)$. A sample of such plots together with their derivatives is shown in Fig. 3. Unfortunately, the plots of Fig. 3(b) show that no clear linear regions are present. This suggests that the branch structure of DLA somehow fails to be self-similar. This is, however, a rather surprising conclusion considering that even a cursory visual inspection of the clusters would seem to suggest otherwise. There is also other convincing direct numerical evidence in the literature that supports the case for the self-similar nature of this branch structure [31-33]. This thus suggests that it is the implementation of the sandbox method that is at fault.

To resolve this issue, we decided to test the method on the object of Fig. 4. This object, which we shall henceforth refer to as the snowflake cluster, possesses $n$-fold symmetry such as radial DLA and was constructed using $\approx 500000$ particles. Its five principal arms were designed to have the same simple 

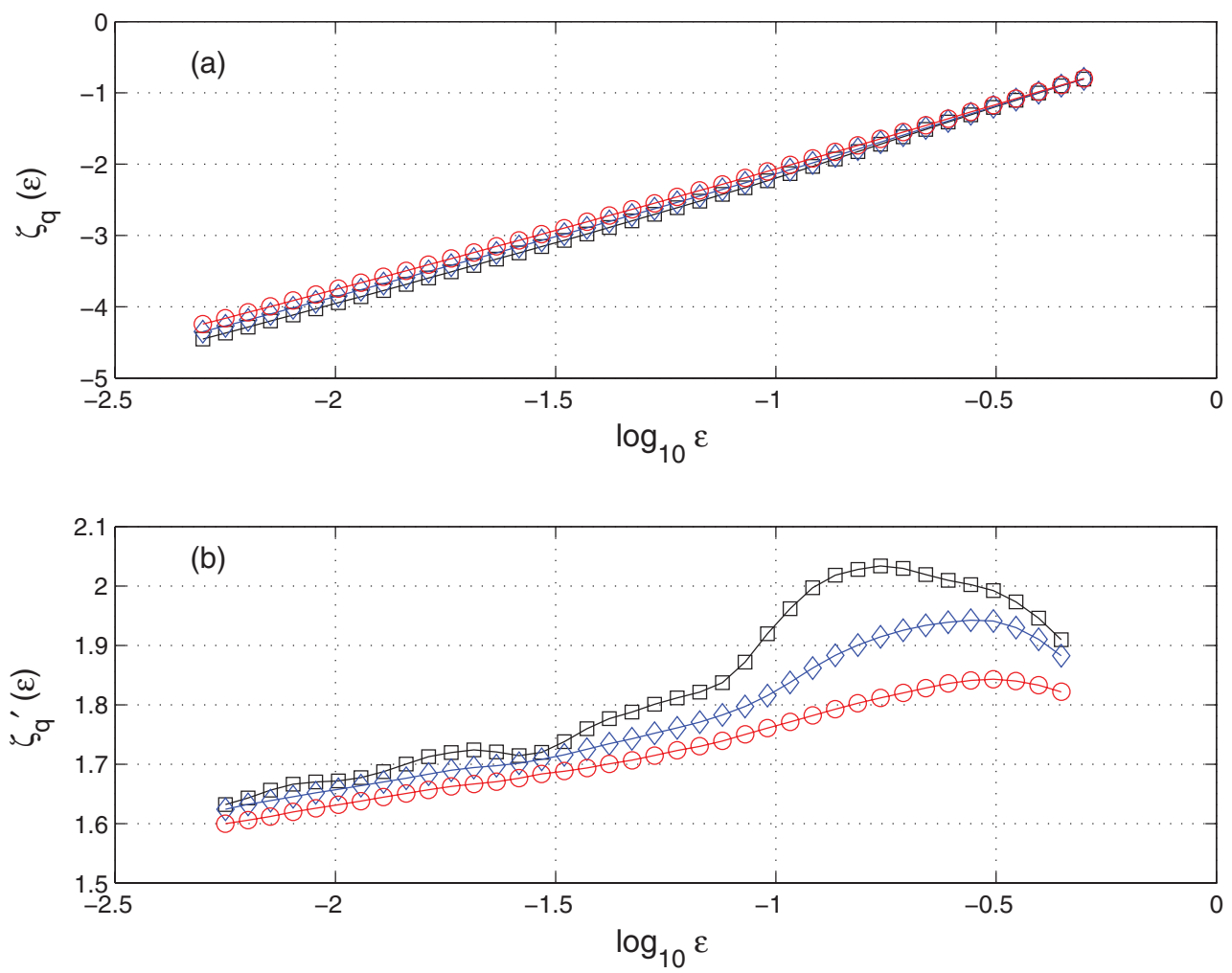

FIG. 3. (Color online) (a) Plots of $\zeta_{q}=\frac{1}{(q-1)}\left\langle\log \left\langle\mu_{\mathbf{b}}^{q-1}\right\rangle\right\rangle$ vs $\log (\epsilon)$ for $q=-3(\square), q=0(\diamond)$, and $q=9$ (o). Data obtained from the frozen zone of 100 DLA clusters of mass $10^{6}$ particles. (b) Derivative of plots shown in (a).

self-similar structure of the Vicsek snowflake fractal of Fig. 5. One would thus expect the $D(q)$ spectrum that characterizes the internal fractal structure of the snowflake cluster to have the trivial $q$-independent form $D(q)=\log 5 / \log 3 \approx$ 1.465 [34].

To obtain a numerical estimate of this $D(q)$, we implemented the sandbox method in a similar fashion to that done previously on the DLA clusters. We thus randomly chose

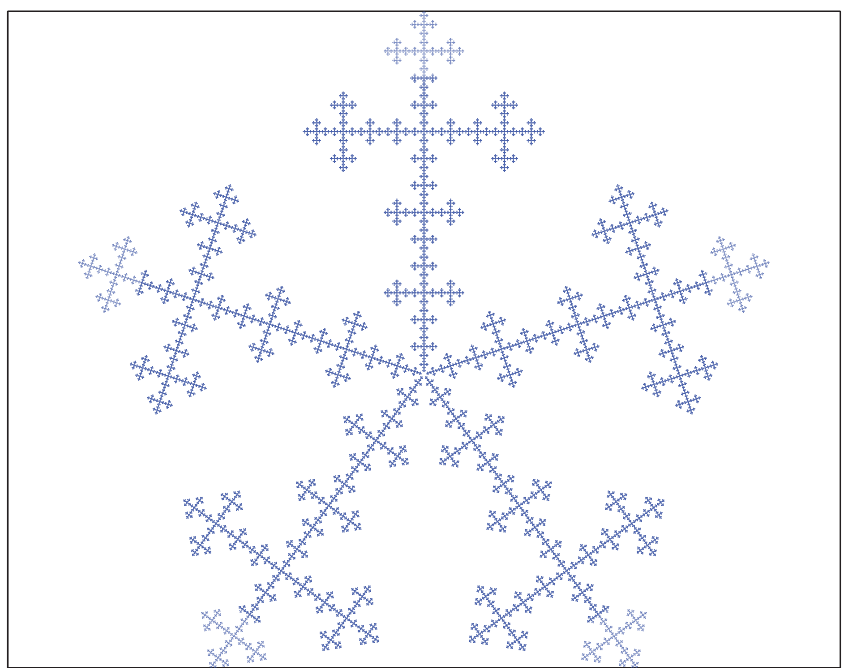

FIG. 4. (Color online) Snowflake cluster. Deterministic fractal model of a radial DLA cluster. It consists of five identical self-similar arms, the structure of each being based on the fractal of Fig. 5 .
10000 reference points from within a distance $0.5 R_{g}$ of the central point. The results are displayed in Fig. 6(a), where we show the numerical derivative of $\zeta_{q}=\frac{1}{(q-1)} \log \left\langle\mu_{\mathbf{b}}^{q-1}\right\rangle$ versus $\log \epsilon$ plots for some selected values of $q$. For all $q$, the derivative oscillates at the lower values of $r$ about the expected value 1.465 , which correctly characterizes the internal fractal structure of the principal arms. However, at larger $r$, we see a prominent and surprising increase in the derivative.

As we know by construction that the internal fractal structure of the object is self-similar, this anomalous scaling phenomena must again be a product of its $n$-fold symmetry. To begin to understand the details as to why this is so, imagine choosing a reference point located at position $\mathbf{b}$ in one of the fractal arms. For small $r$, the sampled measure $\mu_{\mathbf{b}}(r)$ will consist only of points that lie within the same fractal arm and we expect the simple scaling $\mu_{\mathbf{b}}(r) \sim r^{\gamma}$
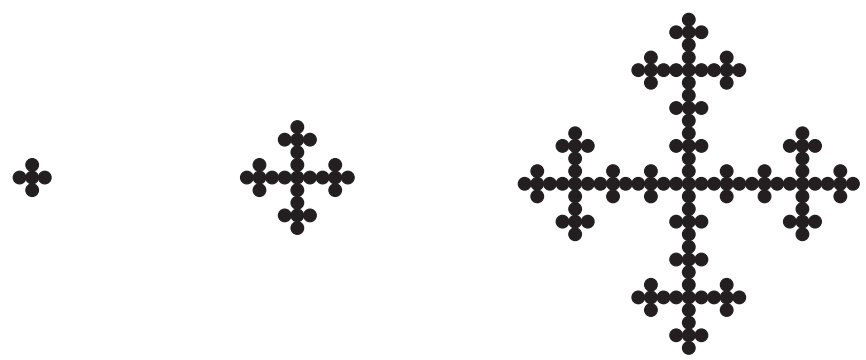

FIG. 5. First three stages in the construction of a snowflake fractal. 

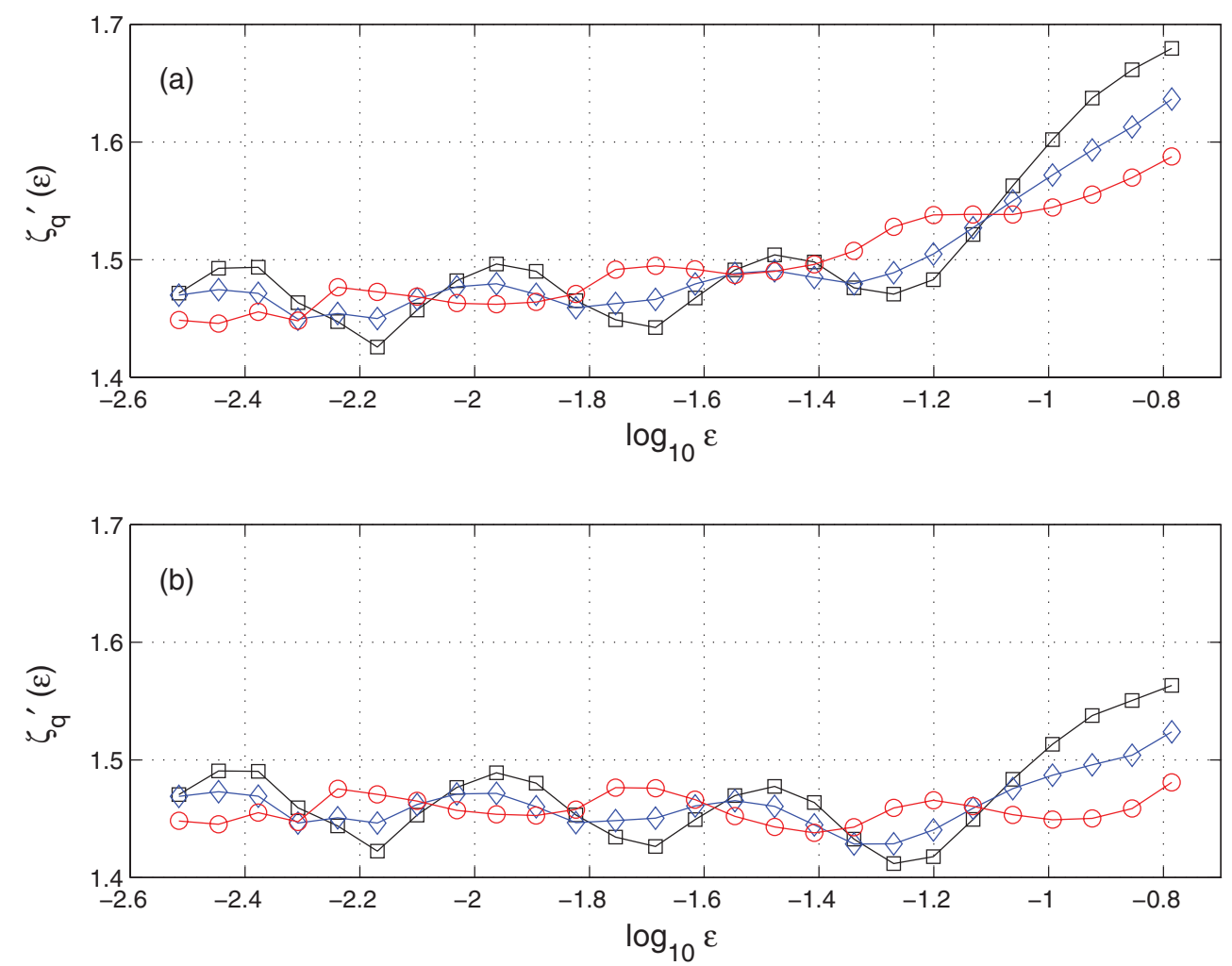

FIG. 6. (Color online) Numerical derivative $\zeta_{q}^{\prime}(\epsilon)$ of $\zeta_{q}=\frac{1}{(q-1)} \log \left\langle\mu_{\mathbf{b}}^{q-1}\right\rangle$ vs $\log (\epsilon)$ plots for $q=-3(\square), q=0(\diamond)$, and $q=9(\bigcirc)$. Data obtained from snowflake cluster of Fig. 4 using reference points, the distance $u=|\mathbf{b}|$ from the central point of which satisfies (a) $u / R_{g}<0.5$ and (b) $0.5<u / R_{g}<1.0$.

with $\gamma=D_{0} \approx 1.465$. Critically, however, as $r$ continues to increase, we begin to sample regions that lie beyond the boundary of the fractal arm. We thus begin to sample empty space, which leads to an artificial decrease in the expected value of $\mu_{\mathbf{b}}(r)$ and a corresponding decrease in the measured exponent $\gamma$. This is a well-known boundary effect and is recognized to lead to the measured values of fractal dimensions being underestimated [35].

More importantly for the present discussion, however, is that, by increasing $r$ further, we begin sampling points that exist within neighboring fractal arms. We now encounter the reverse scenario in that this will instead cause a sudden abnormal increase in $\mu_{\mathbf{b}}(r)$ so that the measured exponent $\gamma$ at large $r$ may thus attain values which exceed $D_{0} \approx 1.465$. It thus seems plausible that the anomalous increase at large $r$ in the plots of Fig. 6(a) is a consequence of this unfortunate sampling of neighboring fractal arms. This is, of course, the very same problem encountered earlier when attempting to accurately measure the lacunarity of DLA clusters. As we did then, we can circumvent this problem somewhat by choosing reference points existing further away from the object's center.

We therefore implemented the sandbox method on the snowflake cluster by choosing only reference points satisfying $0.5 R_{g}<|\mathbf{b}|<R_{g}$. (Note that the central point of the object is located at the origin.) Choosing such reference points ensures that we begin sampling the mass of neighboring fractal arms at much larger values of $r$ than previously. The results are shown in Fig. 6(b). As in Fig. 6(a), we find that the anomalous increase in the derivative of the log-log plots is still present. However, as expected, this increase now begins at a larger value of $r$, so that, critically, we are now able to observe the expected scaling behavior over a much broader range of length scales.

As we shall now show, a similar implementation of the sandbox method performed on DLA clusters will also yield improved results. Recall that our initial multifractal investigation into DLA failed to detect any scaling behavior in the log-log plots of Fig. 3, as evidenced by the steady increase in their derivative. In fact, at large $r$, the derivative climbs to values far in excess of the expected fractal dimension $D_{0} \approx 1.71$ [36]. This is precisely the behavior observed in the snowflake cluster results of Fig. 6(a), which we know to be a numerical artifact caused by the sampling of neighboring cluster arms.

To avoid similar unwanted sampling occurring when analyzing DLA clusters, we thus implemented the sandbox algorithm on our 100 DLA clusters once again, this time selecting only reference points existing in the region $0.5 R_{g}<$ $|\mathbf{b}|<1.5 R_{g}$. The resultant log-log plots are shown in Fig. 7 . For small $r$, the derivative of the $\log$-log plots is, as before, not constant, reflecting the imperfect fractal structure of DLA at these length scales. However, there is now apparent power law scaling over one decade from approximately $\log _{10}\left(r / R_{g}\right)=$ $-1.5 \rightarrow-0.5$. Fitting a line to the $\log$-log plots over this region and taking their slopes, we were subsequently able to estimate the values of $D(q)$ to obtain the spectrum of Fig. 8 . 

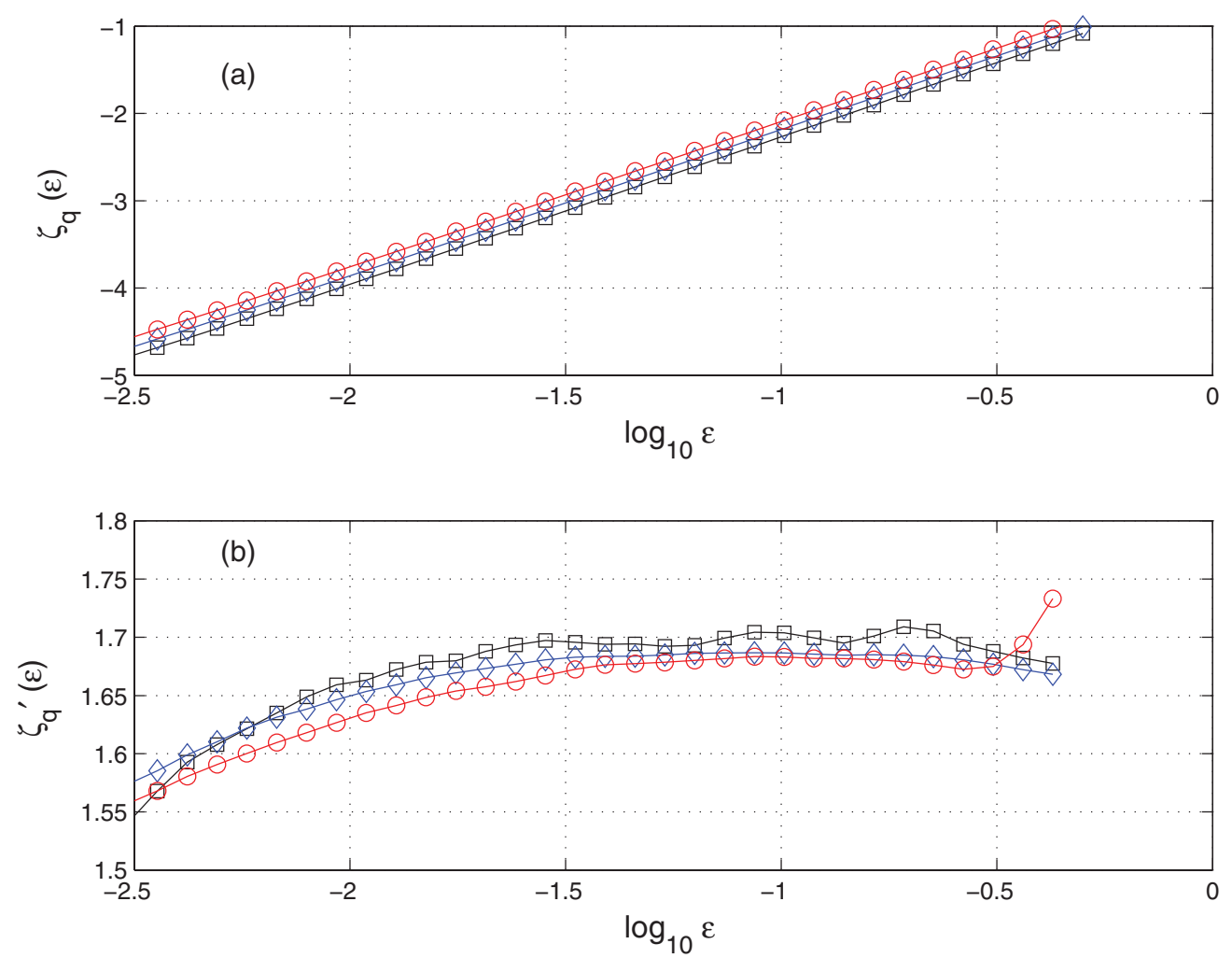

FIG. 7. (Color online) Plots of $\zeta_{q}=\frac{1}{(q-1)}\left\langle\log \left\langle\mu_{\mathbf{b}}^{q-1}\right\rangle\right\rangle$ vs $\log (\epsilon)$ for $q=-3$ ( $\square$ ), $q=0(\diamond)$, and $q=9$ (o). Data obtained from 100 DLA clusters of mass $10^{6}$ particles and using only reference points existing within a distance $0.5 R_{g}<u<1.5 R_{g}$ from the cluster seed point. (b) Derivative $\zeta_{q}^{\prime}$ of plots shown in (a).

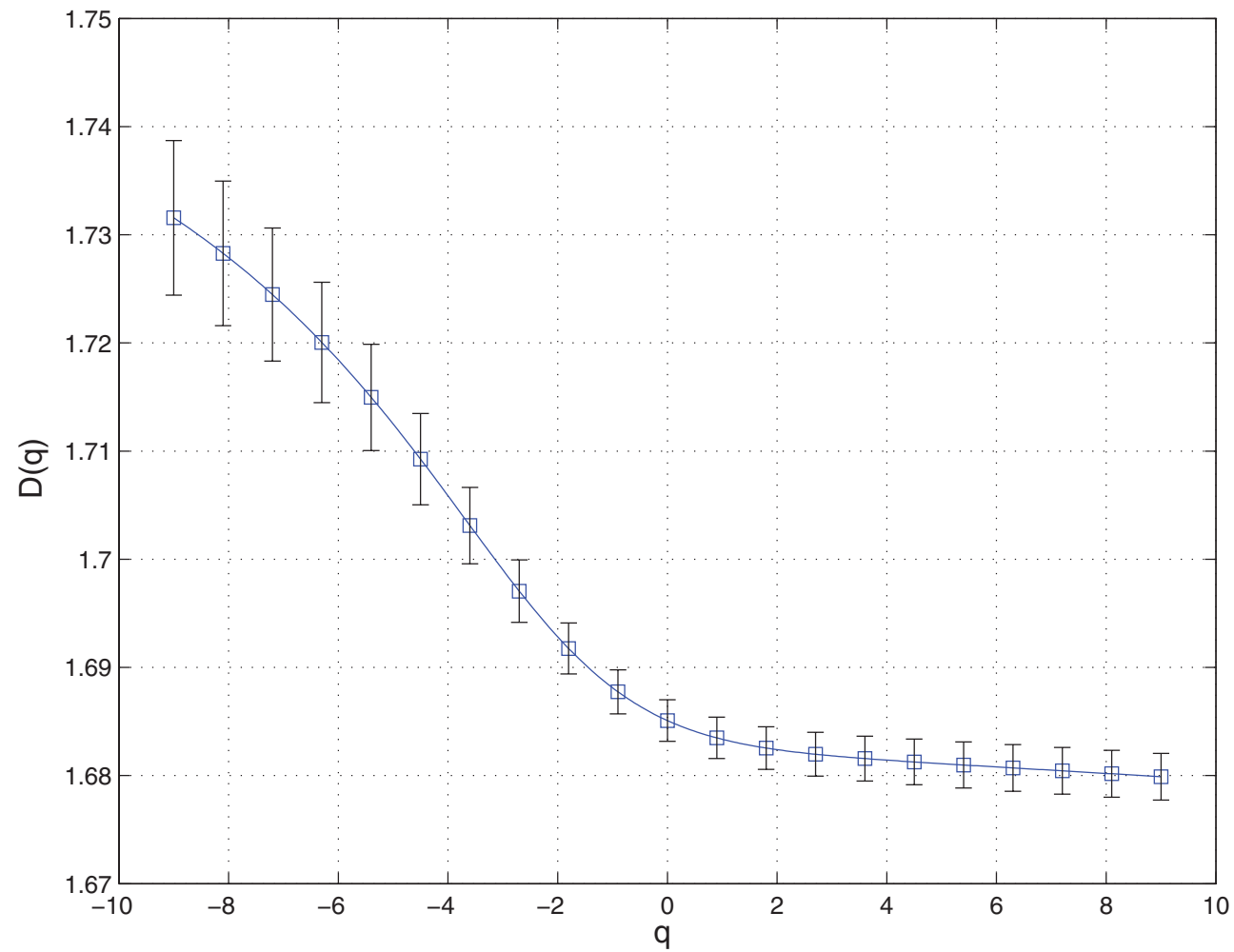

FIG. 8. (Color online) $D(q)$ spectrum of the mass distribution of DLA clusters (100 clusters of $10^{6}$ particles were used). Error bars give $95 \%$ confidence intervals. 


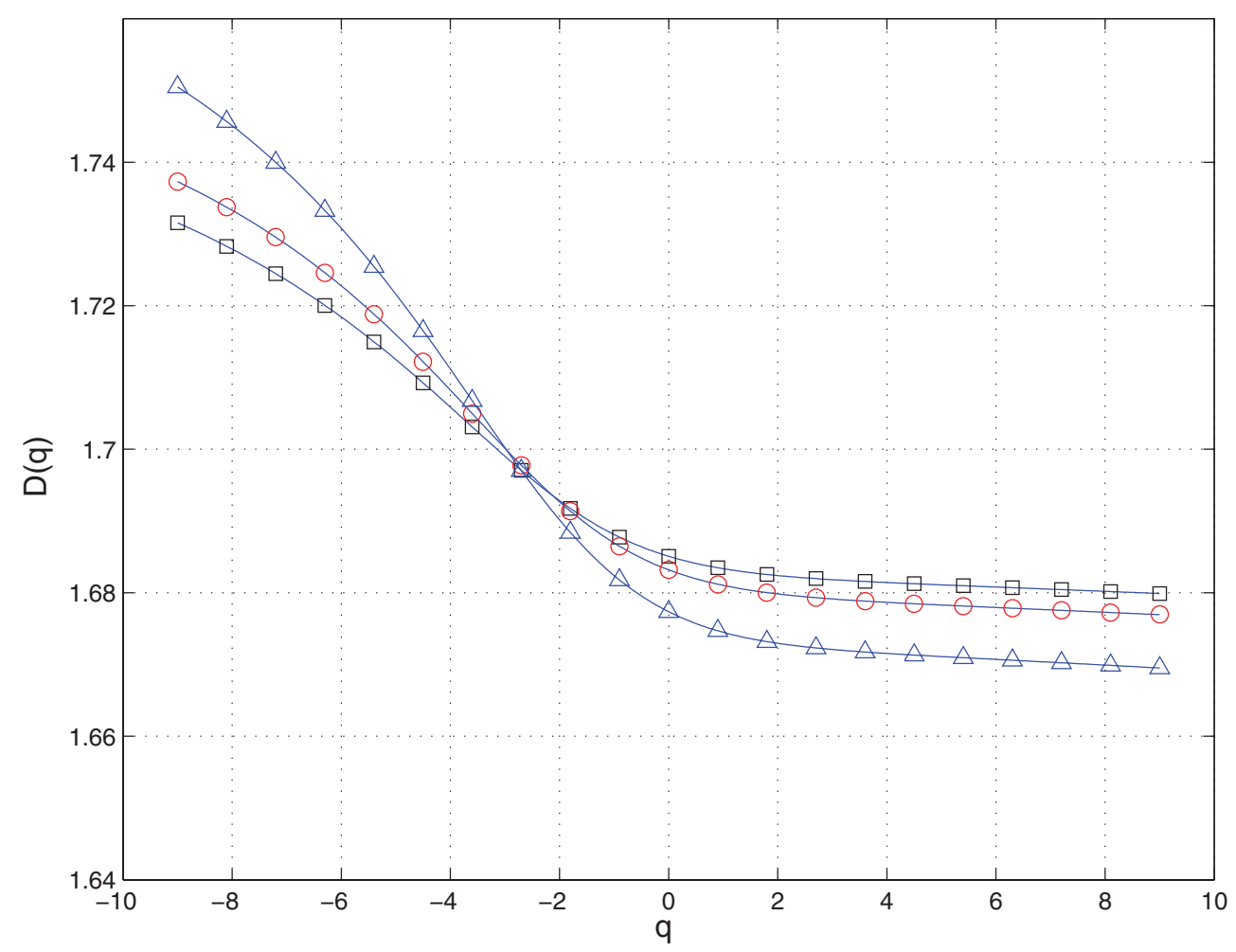

FIG. 9. (Color online) $D(q)$ function calculated from 100 DLA clusters of mass $250000(\triangle), 500000(\circ)$, and $10^{6}(\square)$ particles.

This estimate of $D(q)$ differs markedly to the results obtained previously in an earlier study using the same sandbox technique on identically sized clusters [11]. We in fact find that for all $q$ we obtain $D(q)$ values significantly smaller in magnitude to those found in this earlier work. For example, we found that $D(q=0) \approx 1.685$, while the earlier study yielded $D(0) \approx 1.73$. However, as the reference points were chosen from within the central frozen region of the DLA clusters $\left(|\mathbf{b}|<0.5 R_{g}\right)$, it is our belief that the results quoted in this original study are subject to systematic errors induced by the $n$-fold symmetry of the clusters.

It should also be noted that the value of 1.685 quoted above is merely a lower bound on the fractal dimension $D(0)$. In Fig. 9, we show the $D(q)$ spectrum from Fig. 8 together with similarly obtained estimates calculated from the same 100 DLA clusters at earlier stages in their growth. We find that our results suffer from significant finite-size effects and observe a steady flattening in the estimates of $D(q)$ with increasing cluster mass $M$. In the next section, we discuss the origin of this behavior.

\section{DISCUSSION}

Since the inception of the model, DLA has proved especially difficult to characterize accurately because many of the measurements performed on clusters exhibit strong finite-size effects [18,21,30,32,36-40]. Indeed, we have seen their presence in our own measurements of lacunarity and the spectrum of generalized dimensions $D(q)$. In both cases, we believe that their existence is a product of the randomness (or noise) inherent in DLA. More specifically, we shall argue that they are a consequence of the decrease in the relative noise magnitude with cluster growth $[39,41]$. This is the very same argument put forward by Lam to explain many of the complex scaling hypotheses purported for DLA [41]. In his paper, Lam discusses this idea in some depth, so we shall only give it the briefest of descriptions here.

Let us begin by considering a DLA cluster at two different stages in its growth as shown in Fig. 10. One can clearly see that the branch structure of the younger cluster appears more erratic and noisy than its older self. This demonstrates that, as the cluster grows, the branches become smoother and their general appearance more regular and homogeneous (when viewed at scales comparable to the cluster size $R_{g}$ ). In essence, fluctuations in the branch structure become less pronounced with growth.

This has a direct impact on the measured lacunarity of clusters, which, in effect, quantifies such fluctuations. Given, for example, the noisy irregular structure and the more ordered homogeneous structure of Fig. 11, the lacunarity of the latter at some fixed $r$ will necessarily be lower due to the smaller variability in $\mu_{\mathbf{b}}(r)$. Similarly, at some fixed $\epsilon$, the lacunarity of the older cluster of Fig. 10 will necessarily be smaller than that of its younger self. This thus explains the decrease in $\Phi$ at fixed $\epsilon$ with growth observed in Fig. 2.

In the past, it has been suggested that $\Phi$ may continue this decrease and asymptotically tend to unity [37]. However, there exist both numerical results and sound theoretical arguments to suggest that, as clusters grow larger, the relative noise magnitude decays to some nonzero value $[39,41]$. That is, there will always remain some randomness and inhomogeneity in DLA clusters. This suggests that for fixed $\epsilon$, the $\Phi$ measurements of Fig. 2(b) will decrease to some limiting value greater than unity. 

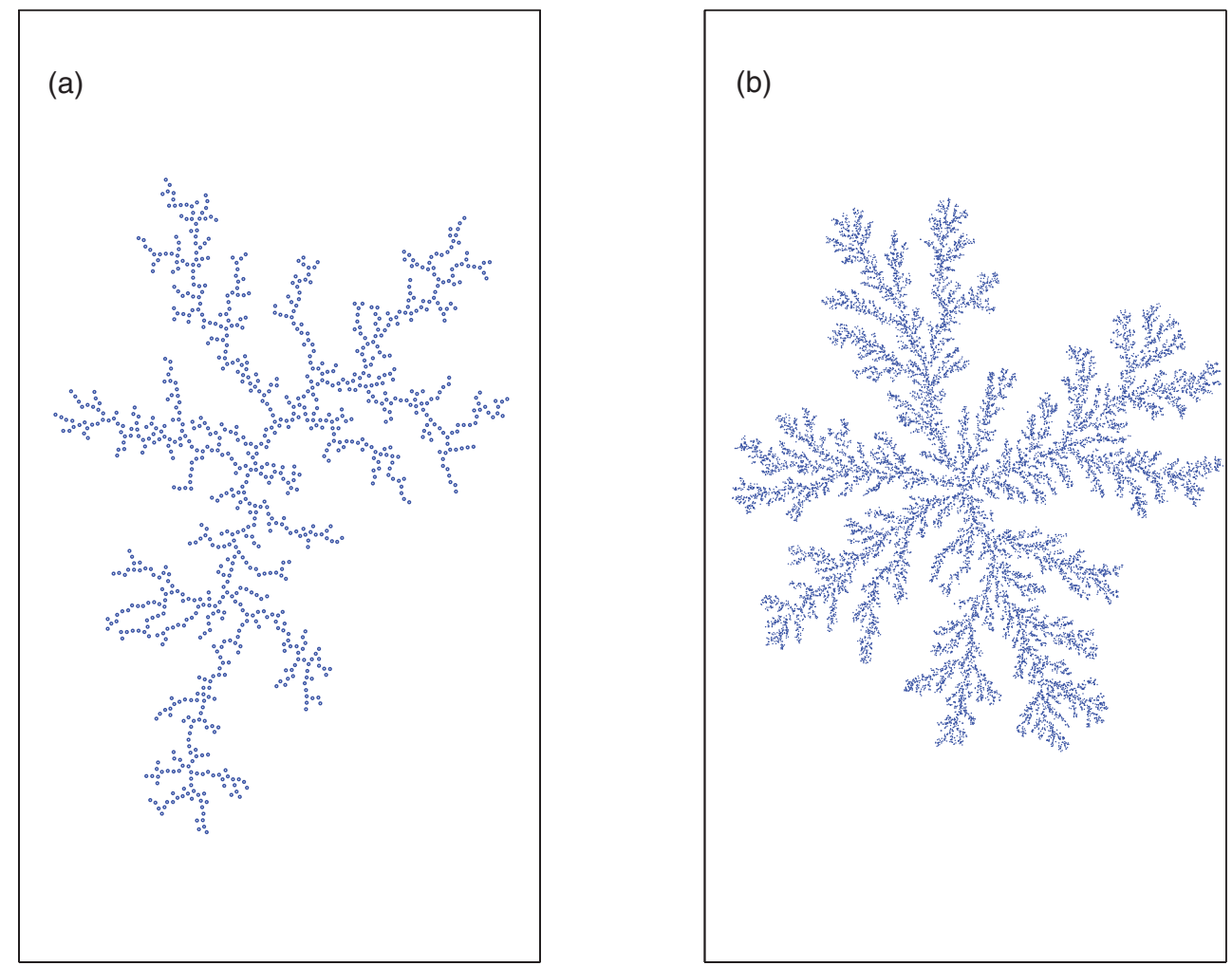

FIG. 10. (Color online) (a) A DLA cluster of mass $M=1000$. (b) The same cluster at mass $M=10^{6}$.

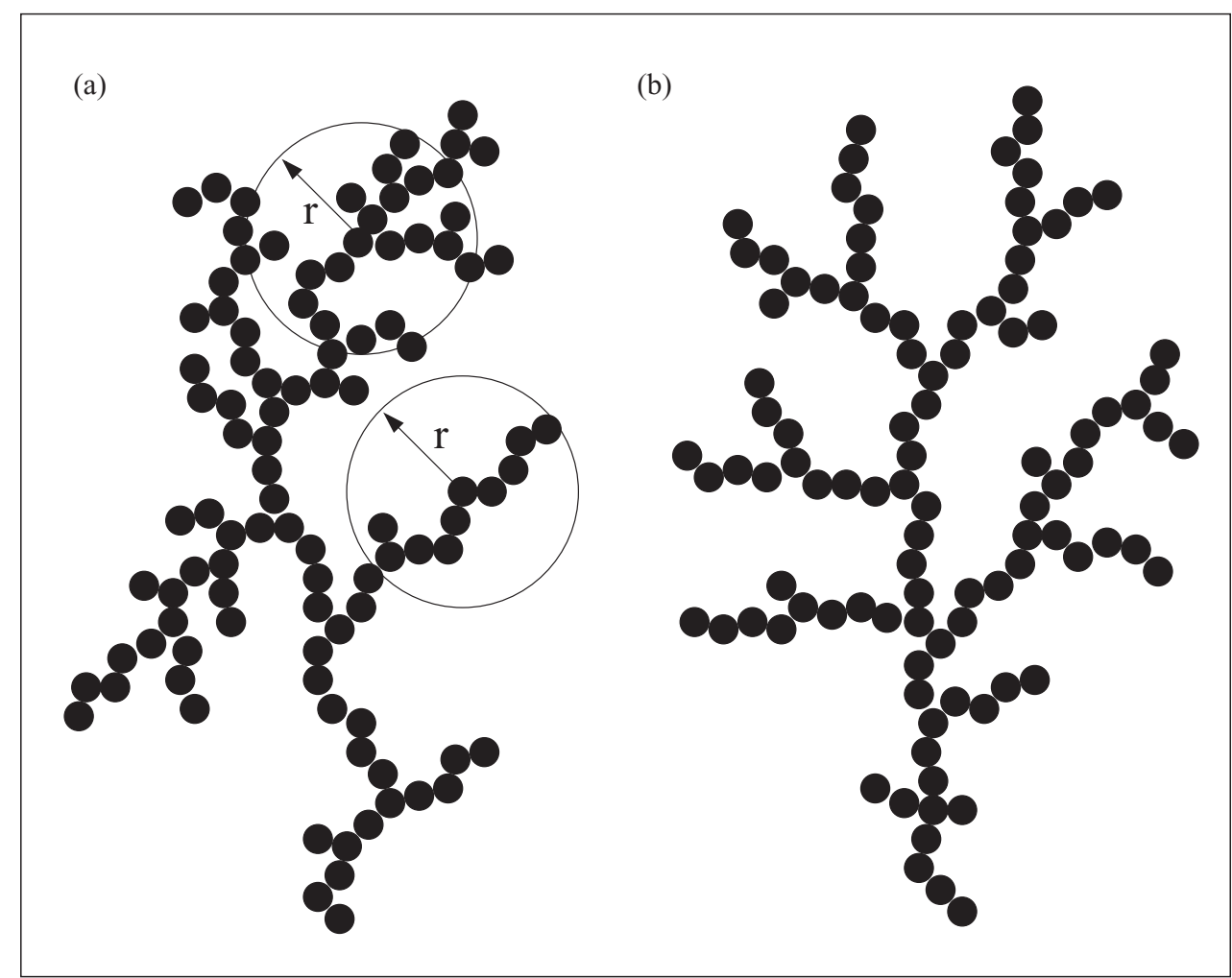

FIG. 11. The variance in $\mu_{\mathbf{b}}(r)$ is greater in the more irregular structure of (a) than that shown in (b). The lacunarity $\Phi(r)$ of the former will thus be consequently larger. 
Other than these finite-size effects in $\Phi$ with growth, Fig. 2 also exhibits another interesting feature. Earlier, we stated that, at a fixed cluster size, $\Phi$ was essentially constant with $r$. However, this is not strictly true. Instead, there does exist a slight decrease in $\Phi$ with $r$ (although the rate of this decrease gets steadily smaller). However, because the clusters appear less noisy and more homogeneous when viewed at larger scales $r$, such a decrease is entirely understandable. The same reasoning can be applied to explain the finite-size effects present in the $D(q)$ estimates of Fig. 9.

Consider a DLA cluster and let us denote by $\mu_{\max }$ and $\mu_{\text {min }}$ the maximal and minimal values of $\mu_{\mathbf{b}}$ at some $\epsilon$. If multifractal, we expect that $\mu_{\max } \sim \epsilon^{D(\infty)}$ and $\mu_{\min } \sim \epsilon^{D(-\infty)}$ so that the ratio

$$
\Lambda(\epsilon)=\frac{\mu_{\max }}{\mu_{\min }} \sim \epsilon^{-\Delta D},
$$

where $\Delta D=D(-\infty)-D(\infty)>0$. Fixing $\epsilon, \Lambda$ should thus be independent of the cluster size.

However, in light of our previous discussion on noise in DLA, one would clearly not expect this to be so. For example, examining the clusters of Fig. 10 at the length scale $\epsilon<1$, we would expect the disparity between $\mu_{\max }$ and $\mu_{\min }$ to be greater for the smaller more inhomogeneous cluster than for the larger cluster. Consequently, for a fixed $\epsilon$, we expect the ratio $\Lambda$ of DLA clusters to decrease with growth.

For Eq. (4) to hold, this immediately implies that $\Delta D$ must also decrease with growth. That is, the expected decrease in the relative noise magnitude with growth manifests itself as the flattening in the $D(q)$ spectrum observed in our results of Fig. 9. At some later stage in the growth of the clusters, however, we expect the relative noise magnitude to stabilize so that for fixed $\epsilon$, both $\Lambda$, and consequently $\Delta D$, become constant with growth. Unfortunately, for the issue of the possible multifractality of DLA, our numerical results say nothing of what this limiting value of $\Delta D$ might be.

However, it is difficult to make a physical case as to why one should expect $\Delta D>0$ asymptotically. For if it was, then $\mu_{\max }$ and $\mu_{\min }$ would scale differently with $\epsilon$, thus implying that there exist disparate regions in the clusters with quite different branch structure. Using the language of branch order analysis, this would require, for example, that either or both of the bifurcation and length ratios should differ with location within the cluster [31-33]. It is hard to see why this should be so.

If we therefore speculate that DLA is indeed monofractal, then Fig. 9 implies that, asymptotically, $D(q) \approx 1.697$ for all $q$, this being the value at which the three finite-size $D(q)$ estimates intersect. This is consistent with the results $D(q)=1.69 \pm 0.03$ and $D(q)=1.70 \pm 0.01$ obtained for positive $q$ in earlier studies $[13,17]$. However, it is highly probable that the value $D(q) \approx 1.697$ is underestimated as a result of the aforementioned boundary effects inherent in the sandbox method [35].

\section{SUMMARY}

The most striking property of DLA clusters is their selfsimilar branch structure. A branch consists of similarly shaped smaller side branches, each of which in turn possesses its own smaller side branches, and so on. However, radial DLA clusters also possess a nonfractal global structure identified by the $n$-fold symmetry of its principal cluster arms. Significantly, this structure is quite distinct from and independent of the fractal branch structure that exists within these arms. Whereas the latter is more fundamental and a product of the diffusion process inherent in the model, the former is principally determined by the geometry of the boundary from which the diffusing particles are released. (For example, launching particles from a line far above a linear substrate, rather than from a circle surrounding a seed point, yields diffusion-limited deposits that lack the $n$-fold symmetry of radial DLA clusters [42].) Conceptually then, it is thus perhaps more useful to think of a radial DLA cluster not as a single fractal object, but rather as a collection of (near identical) fractal objects arranged concentrically about the cluster seed.

In this paper, using standard methods, we measured the lacunarity and $D(q)$ spectrum of the fractal branch structure of radial DLA clusters. We demonstrated how a naive application of these methods can lead to the $n$-fold symmetry inducing anomalous scaling into these measurements. Indeed, such anomalous scaling has, in the past, been mistakenly interpreted as evidence of multifractality in DLA.

However, negating the effects of the $n$-fold symmetry of the clusters, these anomalous scaling features disappear and standard scaling is recovered. Although exhibiting strong finite-size effects, our subsequent estimates of lacunarity $\Phi$ and generalized dimensions $D(q)$ show that the case for multifractality in DLA is not as strong as previously thought. We believe that the finite-size effects in our results reflect the slow decrease in the relative noise magnitude with growth. This argument has previously been put forward as an explanation for finite-size effects exhibited by other measured quantities in DLA [39,41].
[1] T. A. Witten and L. M. Sander, Phys. Rev. Lett. 47, 1400 (1981).

[2] H. E. Stanley, Fractals and Disordered Systems (Springer, Berlin, 1996).

[3] P. Meakin, Fractals, Scaling and Growth Far from Equilibrium (Cambridge University Press, Cambridge, 1998).

[4] P. Meakin, A. Coniglio, H. E. Stanley, and T. A. Witten, Phys. Rev. A 34, 3325 (1986).

[5] T. C. Halsey, P. Meakin, and I. Procaccia, Phys. Rev. Lett. 56, 854 (1986).
[6] C. Amitrano, A. Coniglio, and F. di Liberto, Phys. Rev. Lett. 57, 1016 (1986).

[7] Y. Hayakawa, S. Sato, and M. Matsushita, Phys. Rev. A 36, 1963 (1987).

[8] H. G. E. Hentschel and I. Procaccia, Phys. D (Amsterdam) 8, 435 (1983).

[9] T. C. Halsey, M. H. Jensen, L. P. Kadanoff, I. Procaccia, and B. I. Shraiman, Phys. Rev. A 33, 1141 (1986).

[10] T. Nagatani, Phys. Rev. A 38, 2632 (1988). 
[11] T. Vicsek, F. Family, and P. Meakin, Europhys. Lett. 12, 217 (1990).

[12] F. Argoul, A. Arneodo, G. Grasseau, and H. L. Swinney, Phys. Rev. Lett. 61, 2558 (1988).

[13] G. Li, L. M. Sander, and P. Meakin, Phys. Rev. Lett. 63, 1322 (1989).

[14] F. Argoul, A. Arneodo, J. Elezgaray, G. Grasseau, and R. Murenzi, Phys. Rev. A 41, 5537 (1990).

[15] P. Meakin and S. Havlin, Phys. Rev. A 36, 4428 (1987).

[16] H. Boularot and G. Albinet, Phys. Rev. E 53, 5106 (1996).

[17] W. G. Hanan and D. M. Heffernan, Chaos Solitons Fractals 12 , 193 (2001).

[18] S. Schwarzer, S. Havlin, P. Ossadnik, and H. E. Stanley, Phys. Rev. E 53, 1795 (1996).

[19] T. Tél, A. Fülöp, and T. Vicsek, Phys. A (Amsterdam) 159, 155 (1989).

[20] R. Blumenfeld and B. B. Mandelbrot, Phys. Rev. E 56, 112 (1997).

[21] M. Plischke and Z. Rácz, Phys. Rev. Lett. 53, 415 (1984).

[22] E. P. Rodrigues, M. S. Barbosa, and L. F. Costa, Phys. Rev. E 72, 016707 (2005).

[23] P. Meakin, Phys. D (Amsterdam) 86, 104 (1995).

[24] J. Lee and H. E. Stanley, Phys. Rev. Lett. 61, 2945 (1988).

[25] R. Blumenfeld and A. Aharony, Phys. Rev. Lett. 62, 2977 (1989).
[26] S. Schwarzer, J. Lee, A. Bunde, S. Havlin, H. E. Roman, and H. E. Stanley, Phys. Rev. Lett. 65, 603 (1990).

[27] P. A. Trunfio and P. Alstrøm, Phys. Rev. B 41, 896 (1990).

[28] C. Evertsz, P. Jones, and B. Mandelbrot, J. Phys. A: Math. Gen. 24, 1889 (1991).

[29] M. H. Jensen, A. Levermann, J. Mathiesen, and I. Procaccia, Phys. Rev. E 65, 046109 (2002).

[30] W. G. Hanan and D. M. Heffernan, Phys. Rev. E 77, 011405 (2008).

[31] E. L. Hinrichsen, K. J. Måløy, J. Feder, and T. Jøssang, J. Phys. A: Math. Gen. 22, L271 (1989).

[32] P. Ossadnik, Phys. Rev. A 45, 1058 (1992).

[33] I. Yekutieli, B. B. Mandelbrot, and H. Kaufman, J. Phys. A: Math. Gen. 27, 275 (1994).

[34] T. Vicsek, J. Phys. A: Math. Gen. 16, L647 (1983).

[35] C. Raab and J. Kurths, Phys. Rev. E 64, 016216 (2001).

[36] S. Tolman and P. Meakin, Phys. Rev. A 40, 428 (1989).

[37] B. B. Mandelbrot, Phys. A (Amsterdam) 191, 95 (1992).

[38] C. H. Lam, H. Kaufman, and B. B. Mandelbrot, J. Phys. A: Math. Gen. 28, L213 (1995).

[39] R. C. Ball, N. E. Bowler, L. M. Sander, and E. Somfai, Phys. Rev. E 66, 026109 (2002).

[40] B. B. Mandelbrot, B. Kol, and A. Aharony, Phys. Rev. Lett. 88, 055501 (2002).

[41] C.-H. Lam, Phys. Rev. E 52, 2841 (1995).

[42] P. Meakin, Phys. Rev. A 27, 2616 (1983). 